\title{
A SIEVE M-THEOREM FOR BUNDLED PARAMETERS \\ IN SEMIPARAMETRIC MODELS, WITH APPLICATION \\ TO THE EFFICIENT ESTIMATION IN A LINEAR \\ MODEL FOR CENSORED DATA
}

\section{(Supplementary Material)}

\author{
By YING Ding * AND BIN NAN *,† \\ University of Michigan
}

1. Proofs of technical lemmas. We first prove the lemmas that are needed for the proofs of Theorems 4.1, 4.2 and 4.3.

1.1. Proof of Lemma 7.1. This result follows by direct calculation:

$$
\begin{aligned}
& i_{\beta}(\beta, \zeta(\cdot, \beta) ; Z) \\
& =-X\left\{\Delta \dot{g}\left(\epsilon_{0}-X^{\prime}\left(\beta-\beta_{0}\right)\right)\right. \\
& \left.-\int_{a}^{b} 1\left(\epsilon_{0} \geq t\right) \exp \left\{g\left(t-X^{\prime}\left(\beta-\beta_{0}\right)\right)\right\} \dot{g}\left(t-X^{\prime}\left(\beta-\beta_{0}\right)\right) d t\right\}, \\
& \dot{i}_{\zeta}(\beta, \zeta(\cdot, \beta) ; Z)[h(\cdot, \beta)]=\left.\frac{\partial}{\partial \eta} l(\beta,(\zeta+\eta h)(\cdot, \beta) ; Z)\right|_{\eta=0} \\
& =\Delta w\left(\epsilon_{0}-X^{\prime}\left(\beta-\beta_{0}\right)\right) \\
& -\int_{a}^{b} 1\left(\epsilon_{0} \geq t\right) \exp \left\{g\left(t-X^{\prime}\left(\beta-\beta_{0}\right)\right)\right\} w\left(t-X^{\prime}\left(\beta-\beta_{0}\right)\right) d t, \\
& \ddot{l}_{\beta \beta}(\beta, \zeta(\cdot, \beta) ; Z) \\
& =X X^{\prime}\left\{\Delta \ddot{g}\left(\epsilon_{0}-X^{\prime}\left(\beta-\beta_{0}\right)\right)-\int_{a}^{b} 1\left(\epsilon_{0} \geq t\right) \exp \left\{g\left(t-X^{\prime}\left(\beta-\beta_{0}\right)\right)\right\}\right. \\
& \left.\cdot\left[\ddot{g}\left(t-X^{\prime}\left(\beta-\beta_{0}\right)\right)+\dot{g}^{2}\left(t-X^{\prime}\left(\beta-\beta_{0}\right)\right)\right] d t\right\},
\end{aligned}
$$

\footnotetext{
*Supported in part by NSF Grant DMS-07-06700.

${ }^{\dagger}$ Supported in part by NSF grant DMS-10-07590 and NIH grant R01-AG036802.

AMS 2000 subject classifications: Primary 62E20, 62N01; secondary 62D05

Keywords and phrases: Accelerated failure time model; B-spline; bundled parameters; efficient score function; semiparametric efficiency; sieve maximum likelihood estimation.
} 


$$
\begin{aligned}
\ddot{l}_{\beta \zeta}(\beta, \zeta(\cdot, \beta) ; Z)[h(\cdot, \beta)]=\ddot{l}_{\zeta \beta}^{\prime}(\beta, \zeta(\cdot, \beta) ; Z)[h(\cdot, \beta)] \\
=-X\left\{\Delta \dot{w}\left(\epsilon_{0}-X^{\prime}\left(\beta-\beta_{0}\right)\right)-\int_{a}^{b} 1\left(\epsilon_{0} \geq t\right) \exp \left\{g\left(t-X^{\prime}\left(\beta-\beta_{0}\right)\right)\right\}\right. \\
\left.\cdot\left[\dot{w}\left(t-X^{\prime}\left(\beta-\beta_{0}\right)\right)+\dot{g}\left(t-X^{\prime}\left(\beta-\beta_{0}\right)\right) w\left(t-X^{\prime}\left(\beta-\beta_{0}\right)\right)\right] d t\right\}, \\
\ddot{l}_{\zeta \zeta}(\beta, \zeta(\cdot, \beta) ; Z)\left[h_{1}(\cdot, \beta), h_{2}(\cdot, \beta)\right] \\
=-\int_{a}^{b} 1\left(\epsilon_{0} \geq t\right) \exp \left\{g\left(t-X^{\prime}\left(\beta-\beta_{0}\right)\right)\right\} \\
\quad \cdot w_{1}\left(t-X^{\prime}\left(\beta-\beta_{0}\right)\right) w_{2}\left(t-X^{\prime}\left(\beta-\beta_{0}\right)\right) d t,
\end{aligned}
$$

where $h \in \mathbb{H}=\left\{h: h(\cdot, \beta)=\left.\frac{\partial \zeta_{\eta}(\cdot, \beta)}{\partial \eta}\right|_{\eta=0}=w(\psi(\cdot, \beta)), \zeta_{\eta} \in \mathcal{H}^{p}\right\}$. All the above derivatives are continuous and bounded by Conditions (C.1)-(C.3) and (C.6).

1.2. Proof of Lemma 7.2. This is a direct result of Corollary 6.21 in [2], that is, there exists a $g_{0, n} \in \mathcal{G}_{n}^{p}$ such that $\zeta_{0, n}\left(t, x, \beta_{0}\right)=g_{0, n}(t)$ and

$$
\left\|\zeta_{0, n}\left(\cdot, \beta_{0}\right)-\zeta_{0}\left(\cdot, \beta_{0}\right)\right\|_{\infty}=\left\|g_{0, n}-g_{0}\right\|_{\infty}=O\left(q_{n}^{-p}\right)=O\left(n^{-p \nu}\right) .
$$

1.3. Proof of Lemma 7.3. By the calculation in [3] on page 597, denote the ceiling of $x$ by $\lceil x\rceil$, then for any $\varepsilon>0$, there exists a set of brackets $\left\{\left[g_{i}^{L}, g_{i}^{U}\right]: i=1,2, \cdots,\left\lceil(1 / \varepsilon)^{c_{1} q_{n}}\right\rceil\right\}$ such that for any $g \in \mathcal{G}_{n}^{p}, g_{i}^{L}(t) \leq g(t) \leq$ $g_{i}^{U}(t)$ for some $1 \leq i \leq\left\lceil(1 / \varepsilon)^{c_{1} q_{n}}\right\rceil$ and all $t \in[a, b]$, where $\left\|g_{i}^{U}-g_{i}^{L}\right\|_{\infty} \leq \varepsilon$. Since $\mathcal{B} \subseteq \mathbb{R}^{d}$ is compact, $\mathcal{B}$ can be covered by $\left\lceil c_{2}(1 / \varepsilon)^{d}\right\rceil$ balls with radius $\varepsilon$; that is, for any $\beta \in \mathcal{B}$, there exist $\beta_{s}, 1 \leq s \leq\left\lceil c_{2}(1 / \varepsilon)^{d}\right\rceil$, such that $\left|\beta-\beta_{s}\right| \leq$ $\varepsilon$, i.e., $\left|\left(\beta-\beta_{0}\right)-\left(\beta_{s}-\beta_{0}\right)\right| \leq \varepsilon$, and hence $\left|x^{\prime}\left(\beta-\beta_{0}\right)-x^{\prime}\left(\beta_{s}-\beta_{0}\right)\right| \leq C \varepsilon$ for any $x \in \mathcal{X}$ because of Condition (C.2)(a), where $C>0$ is a constant. This indicates that $t-x^{\prime}\left(\beta-\beta_{0}\right) \in\left[t-x^{\prime}\left(\beta_{s}-\beta_{0}\right)-C \varepsilon, t-x^{\prime}\left(\beta_{s}-\beta_{0}\right)+C \varepsilon\right]$ for any $x$ and $t$. Assume $g_{i}^{L}\left(t-x^{\prime}\left(\beta_{s}-\beta_{0}\right)+c_{1}^{i, t} \varepsilon\right)$ and $g_{i}^{U}\left(t-x^{\prime}\left(\beta_{s}-\beta_{0}\right)+c_{2}^{i, t} \varepsilon\right)$ are the minimum and maximum values of $g_{i}^{L}$ and $g_{i}^{U}$ within the interval $\left[t-x^{\prime}\left(\beta_{s}-\beta_{0}\right)-C \varepsilon, t-x^{\prime}\left(\beta_{s}-\beta_{0}\right)+C \varepsilon\right]$, where $c_{1}^{i, t}$ and $c_{2}^{i, t}$ are two constants that only depend on $g_{i}^{L}, g_{i}^{U}$ and $t$ with $\left|c_{1}^{i, t}\right|,\left|c_{2}^{i, t}\right| \leq C$. So we have

$$
\begin{aligned}
g_{i}^{L}( & \left.-x^{\prime}\left(\beta_{s}-\beta_{0}\right)+c_{1}^{i, t} \varepsilon\right) \leq g_{i}^{L}\left(t-x^{\prime}\left(\beta-\beta_{0}\right)\right) \\
& \leq g\left(t-x^{\prime}\left(\beta-\beta_{0}\right)\right) \leq g_{i}^{U}\left(t-x^{\prime}\left(\beta-\beta_{0}\right)\right) \\
& \leq g_{i}^{U}\left(t-x^{\prime}\left(\beta_{s}-\beta_{0}\right)+c_{2}^{i, t} \varepsilon\right) .
\end{aligned}
$$

Hence we can construct a set of brackets

$$
\left\{\left[m_{i, s}^{L}(Z), m_{i, s}^{U}(Z)\right]: i=1, \cdots,\left\lceil(1 / \varepsilon)^{c_{1} q_{n}}\right\rceil ; s=1, \cdots,\left\lceil c_{2}(1 / \varepsilon)^{d}\right\rceil\right\}
$$


such that for any $m(\theta ; Z) \in \mathcal{F}_{n}$, there exists a pair $(i, s)$ such that for any sample point $Z, m(\theta ; Z) \in\left[m_{i, s}^{L}(Z), m_{i, s}^{U}(Z)\right]$, where

$$
\begin{aligned}
m_{i, s}^{L}(Z)= & \left\{\Delta g_{i}^{L}\left(\epsilon_{0}-X^{\prime}\left(\beta_{s}-\beta_{0}\right)+c_{1}^{i, \epsilon_{0}} \varepsilon\right)\right. \\
& \left.\quad-\int_{a}^{b} 1\left(\epsilon_{0} \geq t\right) \exp \left\{g_{i}^{U}\left(t-x^{\prime}\left(\beta_{s}-\beta_{0}\right)+c_{2}^{i, t} \varepsilon\right)\right\} d t\right\} \\
& -l\left(\theta_{0, n} ; Z\right),
\end{aligned}
$$

and

$$
\begin{aligned}
m_{i, s}^{U}(Z)= & \left\{\Delta g_{i}^{U}\left(\epsilon_{0}-X^{\prime}\left(\beta_{s}-\beta_{0}\right)+c_{2}^{i, \epsilon_{0}} \varepsilon\right)\right. \\
& \left.\quad-\int_{a}^{b} 1\left(\epsilon_{0} \geq t\right) \exp \left\{g_{i}^{L}\left(t-x^{\prime}\left(\beta_{s}-\beta_{0}\right)+c_{1}^{i, t} \varepsilon\right)\right\} d t\right\} \\
& -l\left(\theta_{0, n} ; Z\right) .
\end{aligned}
$$

It then follows that

$$
\begin{aligned}
& \left|m_{i, s}^{U}(Z)-m_{i, s}^{L}(Z)\right| \\
& \leq\left|g_{i}^{U}\left(\epsilon_{0}-X^{\prime}\left(\beta_{s}-\beta_{0}\right)+c_{2}^{i, \epsilon_{0}} \varepsilon\right)-g_{i}^{L}\left(\epsilon_{0}-X^{\prime}\left(\beta_{s}-\beta_{0}\right)+c_{1}^{i, \epsilon_{0}} \varepsilon\right)\right| \\
& \quad+\int_{a}^{b} \mid \exp \left\{g_{i}^{U}\left(t-X^{\prime}\left(\beta_{s}-\beta_{0}\right)+c_{2}^{i, t} \varepsilon\right)\right\} \\
& \quad-\exp \left\{g_{i}^{L}\left(t-x^{\prime}\left(\beta_{s}-\beta_{0}\right)+c_{1}^{i, t} \varepsilon\right)\right\} \mid d t \\
& \quad=A_{1}+A_{2} .
\end{aligned}
$$

For $A_{1}$, by subtracting and adding the terms $g\left(\epsilon_{0}-X^{\prime}\left(\beta_{s}-\beta_{0}\right)+c_{2}^{i, \epsilon_{0}} \varepsilon\right)$ and $g\left(\epsilon_{0}-X^{\prime}\left(\beta_{s}-\beta_{0}\right)+c_{1}^{i, \epsilon_{0}} \varepsilon\right)$ and applying the Taylor expansion to $g$ at $\epsilon_{0}-X^{\prime}\left(\beta_{s}-\beta_{0}\right)+c_{1}^{i, \epsilon_{0}} \varepsilon$, we have

$$
\begin{aligned}
A_{1} \leq & \left|g_{i}^{U}\left(\epsilon_{0}-X^{\prime}\left(\beta_{s}-\beta_{0}\right)+c_{2}^{i, \epsilon_{0}} \varepsilon\right)-g\left(\epsilon_{0}-X^{\prime}\left(\beta_{s}-\beta_{0}\right)+c_{2}^{i, \epsilon_{0}} \varepsilon\right)\right| \\
& \quad+\left|g\left(\epsilon_{0}-X^{\prime}\left(\beta_{s}-\beta_{0}\right)+c_{2}^{i, \epsilon_{0}} \varepsilon\right)-g\left(\epsilon_{0}-X^{\prime}\left(\beta_{s}-\beta_{0}\right)+c_{1}^{i, \epsilon_{0}} \varepsilon\right)\right| \\
& \quad+\left|g\left(\epsilon_{0}-X^{\prime}\left(\beta_{s}-\beta_{0}\right)+c_{1}^{i, \epsilon_{0}} \varepsilon\right)-g_{i}^{L}\left(\epsilon_{0}-X^{\prime}\left(\beta_{s}-\beta_{0}\right)+c_{1}^{i, \epsilon_{0}} \varepsilon\right)\right| \\
\leq & \left\|g_{i}^{U}-g\right\|_{\infty}+\left|\dot{g}\left(\epsilon_{0}-X^{\prime}\left(\beta_{s}-\beta_{0}\right)+\tilde{c} \varepsilon\right)\left(c_{2}^{i, \epsilon_{0}}-c_{1}^{i, \epsilon_{0}}\right) \varepsilon\right|+\left\|g-g_{i}^{L}\right\|_{\infty} \\
\leq & \left\|g_{i}^{U}-g_{i}^{L}\right\|_{\infty}+C_{1}\left|\left(c_{2}^{i, \epsilon_{0}}-c_{1}^{i, \epsilon_{0}}\right)\right| \varepsilon+\left\|g_{i}^{U}-g_{i}^{L}\right\|_{\infty} \\
\leq & 2 \varepsilon+2 C_{1} C_{2} \varepsilon \lesssim \varepsilon,
\end{aligned}
$$

where the third inequality holds because $\left\|g_{i}^{U}-g\right\|_{\infty},\left\|g-g_{i}^{L}\right\|_{\infty} \leq\left\|g_{i}^{U}-g_{i}^{L}\right\|_{\infty}$ and $\dot{g}$ is bounded by $C_{1}$. The Constant $C_{1}$ may be proportional to $c_{n}$ that 
is allowed to grow with $n$ slowly enough, but it does not affect the later calculations on convergence rate (see [3], page 591, for their constant $l_{n}$ ), thus we drop $c_{n}$ for simplicity. For $A_{2}$, by using the similar arguments as for $A_{1}$ and denote $t-X^{\prime}\left(\beta_{s}-\beta_{0}\right)=t_{s}$ for notational simplicity, we have

$$
\begin{aligned}
A_{2} \leq \int_{a}^{b} & \left\{\left|\exp \left\{g_{i}^{U}\left(t_{s}+c_{2}^{i, t} \varepsilon\right)\right\}-\exp \left\{g\left(t_{s}+c_{2}^{i, t} \varepsilon\right)\right\}\right|\right. \\
& +\left|\exp \left\{g\left(t_{s}+c_{2}^{i, t} \varepsilon\right)\right\}-\exp \left\{g\left(t_{s}+c_{1}^{i, t} \varepsilon\right)\right\}\right| \\
& \left.+\left|\exp \left\{g\left(t_{s}+c_{1}^{i, t} \varepsilon\right)\right\}-\exp \left\{g_{i}^{L}\left(t_{s}+c_{1}^{i, t} \varepsilon\right)\right\}\right|\right\} d t \\
=\quad \int_{a}^{b} & \left\{\left|\exp \left\{\tilde{g}_{i}^{U}\left(t_{s}+c_{2}^{i, t} \varepsilon\right)\right\}\left(g_{i}^{U}-g\right)\left(t_{s}+c_{2}^{i, t} \varepsilon\right)\right|\right. \\
& +\left|\exp \left\{g\left(t_{s}+\tilde{c} \varepsilon\right)\right\}\left(c_{2}^{i, t}-c_{1}^{i, t}\right) \varepsilon\right| \\
& \left.+\left|\exp \left\{\tilde{g}_{i}^{L}\left(t_{s}+c_{1}^{i, t} \varepsilon\right)\right\}\left(g_{i}^{L}-g\right)\left(t_{s}+c_{1}^{i, t} \varepsilon\right)\right|\right\} d t \\
\lesssim \| & g_{i}^{U}-g\left\|_{\infty}+\left|\left(c_{2}^{i, t}-c_{1}^{i, t}\right) \varepsilon\right|+\right\| g-g_{i}^{L} \|_{\infty} \lesssim \varepsilon .
\end{aligned}
$$

The above equality is from Taylor expansion, where $\tilde{g}_{i}^{U}=g+\xi\left(g_{i}^{U}-g\right)$ for some $0<\xi<1$ and thus $\left|\tilde{g}_{i}^{U}(\cdot)\right| \leq|g(\cdot)|+\varepsilon$, which is bounded in $[a, b]$; similarly $\left|\tilde{g}_{i}^{L}\right|$ is also bounded in $[a, b]$. Hence $\left\|m_{i}^{U}-m_{i}^{L}\right\|_{\infty} \lesssim \varepsilon$ and the $\varepsilon$-bracketing number associated with $\|\cdot\|_{\infty}$ norm for the class $\mathcal{F}_{n}$ follows

$$
N_{[]}\left(\varepsilon, \mathcal{F}_{n},\|\cdot\|_{\infty}\right) \leq(1 / \varepsilon)^{c_{1} q_{n}} c_{2}(1 / \varepsilon)^{d} \lesssim(1 / \varepsilon)^{c_{1} q_{n}+d} .
$$

1.4. Proof of Lemma 7.4. In the proof of Theorem 4.2 in the main text of the paper we show that such defined $\left(h_{1}^{*}, \ldots, h_{d}^{*}\right)$ determines the least favorable submodel for $\beta$. Now, by Conditions (C.4)-(C.5), the following conditional density of $\epsilon_{0}$ given $X$

$$
f_{\epsilon_{0} \mid X}(t \mid X=x)=f(t) \bar{G}_{C \mid X}\left(t+x^{\prime} \beta_{0} \mid X=x\right)+g_{C \mid X}\left(t+x^{\prime} \beta_{0} \mid X=x\right) \bar{F}(t)
$$

is uniformly bounded for all $x \in \mathcal{X}$, and its derivative with respect to $t$

$$
\begin{aligned}
& \dot{f}_{\varepsilon_{0} \mid X}(t \mid X=x) \\
& =\dot{f}(t) \bar{G}_{C \mid X}\left(t+x^{\prime} \beta_{0} \mid X=x\right)-f(t) g_{C \mid X}\left(t+x^{\prime} \beta_{0} \mid X=x\right) \\
& \quad+\dot{g}_{C \mid X}\left(t+x^{\prime} \beta_{0} \mid X=x\right) \bar{F}(t)-g_{C \mid X}\left(t+x^{\prime} \beta_{0} \mid X=x\right) f(t)
\end{aligned}
$$

is also uniformly bounded. Hence the density of $\epsilon_{0}$

$$
f_{\epsilon_{0}}(t)=\int_{\mathcal{X}} f_{\epsilon_{0} \mid X}(t \mid X=x) f_{X}(x) d x
$$


and its derivative

$$
\dot{f}_{\epsilon_{0}}(t)=\int_{\mathcal{X}} \dot{f}_{\epsilon_{0} \mid X}(t \mid X=x) f_{X}(x) d x
$$

are bounded. Thus the first and second derivatives of $P\left(\epsilon_{0} \geq t\right)$, i.e., $-f_{\epsilon_{0}}(t)$ and $-\dot{f}_{\epsilon_{0}}(t)$, are both bounded. In addition, under Condition (C.2)(a), the first and second derivatives of $P\left[X 1\left(\epsilon_{0} \geq t\right)\right]$ with respect to $t$

$$
\frac{d P\left[X 1\left(\epsilon_{0} \geq t\right)\right]}{d t}=-\int_{\mathcal{X}} x f_{X}(x) f_{\epsilon_{0} \mid X}(t \mid X=x) d x
$$

and

$$
\frac{d^{2} P\left[X 1\left(\epsilon_{0} \geq t\right)\right]}{d t^{2}}=-\int_{\mathcal{X}} x f_{X}(x) \dot{f}_{\epsilon_{0} \mid X}(t \mid X=x) d x
$$

are also bounded. Therefore, $P\left[X \mid \epsilon_{0} \geq t\right]=P\left[X 1\left(\epsilon_{0} \geq t\right)\right] / P\left(\epsilon_{0} \geq t\right)$ has a bounded second derivative with respect to $t$ for $t \leq \tau$, where $\tau$ is the truncation time defined in Condition (C.3). Thus $P\left[X \mid \epsilon_{0} \geq t\right] \in \mathcal{G}^{2}$. Moreover, since $g_{0} \in \mathcal{G}^{p}$ for $p \geq 3$, we have $\dot{g}_{0} \in \mathcal{G}^{p-1}$ with $p-1 \geq 2$. Thus according to Corollary 6.21 of [2], there exists an $h_{j, n}^{*} \in \mathcal{H}_{n}^{\min (p-1,2)}=\mathcal{H}_{n}^{2}$ such that $h_{j, n}^{*}\left(t, x, \beta_{0}\right)=w_{j, n}^{*}\left(\psi\left(t, x, \beta_{0}\right)\right)=w_{j, n}^{*}(t)$ and $\left\|h_{j, n}^{*}\left(\cdot, \beta_{0}\right)-h_{j}^{*}\left(\cdot, \beta_{0}\right)\right\|_{\infty}=$ $\left\|w_{j, n}^{*}-w_{j}^{*}\right\|_{\infty}=O\left(q_{n}^{-2}\right)=O\left(n^{-2 \nu}\right)$.

1.5. Proof of Lemma 7.5. The proof is similar to the bracketing number calculation in Lemma 7.3, thus omitted. We refer all the details to [1].

1.6. Proof of Lemma 7.6. The proof is also similar to the bracketing number calculation in Lemma 7.3, thus omitted. We again refer all the details to $[1]$.

2. Proof of Theorem 4.1. We shall apply Theorem 1 of [3] to derive the convergence rate. We proceed by verifying their conditions $\mathrm{C} 1-\mathrm{C} 3$. Since $\operatorname{Pl}(\beta, \zeta(\cdot, \beta) ; Z)$ is maximized at $\left(\beta_{0}, \zeta_{0}\left(\cdot, \beta_{0}\right)\right)$, its first derivatives at $\left(\beta_{0}, \zeta_{0}\left(\cdot, \beta_{0}\right)\right)$ are equal to 0 . By Lemma 7.1 that all the second derivatives of $l(\beta, \zeta(\cdot, \beta) ; Z)$ are continuous and bounded, the Taylor expansion yields

$$
\begin{aligned}
P l(\beta, \zeta(\cdot, \beta) ; Z)-P l\left(\beta_{0}, \zeta_{0}\left(\cdot, \beta_{0}\right) ; Z\right) & =\frac{1}{2} P\left\{\left(\beta-\beta_{0}\right)^{\prime} \ddot{l}_{\beta \beta}\left(\beta_{0}, \zeta_{0}\left(\cdot, \beta_{0}\right) ; Z\right)\left(\beta-\beta_{0}\right)\right. \\
& +2\left(\beta-\beta_{0}\right)^{\prime} \ddot{l}_{\beta \zeta}\left(\beta_{0}, \zeta_{0}\left(\cdot, \beta_{0}\right) ; Z\right)\left[\zeta(\cdot, \beta)-\zeta_{0}\left(\cdot, \beta_{0}\right)\right] \\
& \left.+\ddot{l}_{\zeta \zeta}\left(\beta_{0}, \zeta_{0}\left(\cdot, \beta_{0}\right) ; Z\right)\left[\zeta(\cdot, \beta)-\zeta_{0}\left(\cdot, \beta_{0}\right), \zeta(\cdot, \beta)-\zeta_{0}\left(\cdot, \beta_{0}\right)\right]\right\} \\
& +o\left(d^{2}\left(\theta, \theta_{0}\right)\right) \\
=A & +o\left(d^{2}\left(\theta, \theta_{0}\right)\right),
\end{aligned}
$$


where $\theta=(\beta, \zeta(\cdot, \beta)) \in \Theta_{n}^{p}$. By the model assumption, we have that the conditional expectation $P\left\{i_{\zeta}\left(\beta_{0}, \zeta_{0}\left(\cdot, \beta_{0}\right) ; Z\right)\left[h\left(\cdot, \beta_{0}\right)\right] \mid X\right\}=0$ for all $h \in \mathbb{H}$. Taking $h$ to be $\ddot{g}_{0}$ and $\dot{g}(\psi(\cdot, \beta))-\dot{g}_{0}\left(\psi\left(\cdot, \beta_{0}\right)\right)$ respectively, we have

$$
P\left\{\Delta \ddot{g}_{0}\left(\epsilon_{0}\right)-\int_{a}^{b} 1\left(\epsilon_{0} \geq t\right) \exp \left\{g_{0}(t)\right\} \ddot{g}_{0}(t) d t \mid X\right\}=0
$$

and

$$
\begin{aligned}
P\{ & \Delta\left[\dot{g}\left(\epsilon_{0}-X^{\prime}\left(\beta-\beta_{0}\right)\right)-\dot{g}_{0}\left(\epsilon_{0}\right)\right] \\
& \left.-\int_{a}^{b} 1\left(\epsilon_{0} \geq t\right) \exp \left\{g_{0}(t)\right\}\left[\dot{g}\left(t-X^{\prime}\left(\beta-\beta_{0}\right)\right)-\dot{g}_{0}(t)\right] d t \mid X\right\}=0 .
\end{aligned}
$$

Then it follows

$$
\begin{aligned}
A=P\{ & \frac{1}{2} \int_{a}^{b} 1\left(\epsilon_{0} \geq t\right) \exp \left\{g_{0}(t)\right\}\left\{-\left[\dot{g}_{0}(t) X^{\prime}\left(\beta-\beta_{0}\right)\right]^{2}\right. \\
& +2 \dot{g}_{0}(t) X^{\prime}\left(\beta-\beta_{0}\right)\left[g\left(t-X^{\prime}\left(\beta-\beta_{0}\right)\right)-g_{0}(t)\right] \\
& \left.\left.-\left[g\left(t-X^{\prime}\left(\beta-\beta_{0}\right)\right)-g_{0}(t)\right]^{2}\right\} d t\right\} \\
=-\frac{1}{2} \int_{a}^{b} & \exp \left\{g_{0}(t)\right\} P\left\{1 ( \epsilon _ { 0 } \geq t ) \left[\dot{g}_{0}(t) X^{\prime}\left(\beta-\beta_{0}\right)\right.\right. \\
- & \left.\left.\left(g\left(t_{\beta}\right)-g_{0}(t)\right)\right]^{2}\right\} d t,
\end{aligned}
$$

where $t_{\beta}=t-X^{\prime}\left(\beta-\beta_{0}\right)$. The integrand is from $a$ to $\tau=b$ because of Condition (C.3) and (C.6). Denote $s_{0}(t)=-\dot{g}_{0}(t), s_{1}\left(t ; \epsilon_{0}, X\right)=1\left(\epsilon_{0} \geq\right.$ t) $X^{\prime}\left(\beta-\beta_{0}\right)$, and $s_{2}\left(t ; \epsilon_{0}, X\right)=1\left(\epsilon_{0} \geq t\right)\left[g\left(t_{\beta}\right)-g_{0}(t)\right]$, then

$$
\begin{aligned}
& P\left\{1\left(\epsilon_{0} \geq t\right)\left[\dot{g}_{0}(t) X^{\prime}\left(\beta-\beta_{0}\right)-\left(g\left(t_{\beta}\right)-g_{0}(t)\right)\right]^{2}\right\} \\
& =P\left\{\left[s_{0}(t) s_{1}\left(t ; \epsilon_{0}, X\right)+s_{2}\left(t ; \epsilon_{0}, X\right)\right]^{2}\right\} \\
& \geq s_{0}^{2}(t) P\left[s_{1}^{2}\left(t ; \epsilon_{0}, X\right)\right]+P\left[s_{2}^{2}\left(t ; \epsilon_{0}, X\right)\right] \\
& \quad \quad-2\left|s_{0}(t) P\left[s_{1}\left(t ; \epsilon_{0}, X\right) s_{2}\left(t ; \epsilon_{0}, X\right)\right]\right| \\
& \geq s_{0}^{2}(t) P\left[s_{1}^{2}\left(t ; \epsilon_{0}, X\right)\right]+P\left[s_{2}^{2}\left(t ; \epsilon_{0}, X\right)\right] \\
& \quad-(1-\eta)^{\frac{1}{2}} \cdot 2\left|s_{0}(t)\left[P\left(s_{1}^{2}\left(t ; \epsilon_{0}, X\right)\right)\right]^{\frac{1}{2}}\right| \cdot\left|\left[P\left(s_{2}^{2}\left(t ; \epsilon_{0}, X\right)\right)\right]^{\frac{1}{2}}\right| \\
& \geq\left\{1-(1-\eta)^{\frac{1}{2}}\right\}\left\{s_{0}^{2}(t) P\left(s_{1}^{2}\left(t ; \epsilon_{0}, X\right)\right)+P\left(s_{2}^{2}\left(t ; \epsilon_{0}, X\right)\right)\right\} \\
& \geq \quad \dot{g}_{0}^{2}(t)\left(\beta-\beta_{0}\right)^{\prime} P\left[1\left(\epsilon_{0} \geq t\right) X X^{\prime}\right]\left(\beta-\beta_{0}\right) \\
& \quad+P\left[1\left(\epsilon_{0} \geq t\right)\left(g\left(t_{\beta}\right)-g_{0}(t)\right)^{2}\right],
\end{aligned}
$$


where (2.3) is obtained by using the same argument in [4] on page 2126, which is, under Condition (C.7), $\left[P\left(s_{1} s_{2}\right)\right]^{2} \leq(1-\eta) P\left(s_{1}^{2}\right) P\left(s_{2}^{2}\right)$ for some $\eta \in(0,1)$. Hence from $(2.2)$ we have

$$
\begin{aligned}
A \lesssim & -\left\{\left(\beta-\beta_{0}\right)^{\prime}\left[\int_{a}^{b} \exp \left\{g_{0}(t)\right\} \dot{g}_{0}^{2}(t) P\left[1\left(\epsilon_{0} \geq t\right) X X^{\prime}\right] d t\right]\left(\beta-\beta_{0}\right)\right. \\
& \left.+\int_{a}^{b} \exp \left\{g_{0}(t)\right\} P\left[1\left(\epsilon_{0} \geq t\right)\left(g\left(t_{\beta}\right)-g_{0}(t)\right)^{2}\right] d t\right\} \\
= & -\left(A_{1}+A_{2}\right) .
\end{aligned}
$$

For $A_{1}$, Condition (C.3) implies that

$$
P\left[1\left(\epsilon_{0} \geq t\right) X X^{\prime}\right]=P\left[X X^{\prime} P\left(\epsilon_{0} \geq t \mid X\right)\right] \geq P\left[X X^{\prime} P\left(\epsilon_{0} \geq \tau \mid X\right)\right] \geq \delta P\left(X X^{\prime}\right) .
$$

Then Condition (C.2)(b) yields that $P\left(X X^{\prime}\right)$ is positive definite and thus its smallest eigenvalue $\lambda_{1}>0$. In addition, $\int_{a}^{b} \exp \left\{g_{0}(t)\right\} \dot{g}_{0}^{2}(t) d t$ is bounded away from zero since $\exp \left\{g_{0}(t)\right\}, \dot{g}_{0}^{2}(t) \geq 0$ but not a constant zero on $t \in$ $[a, b]$. Hence it follows that

$$
A_{1} \gtrsim\left(\beta-\beta_{0}\right)^{\prime} P\left(X X^{\prime}\right)\left(\beta-\beta_{0}\right) \geq \lambda_{1}\left|\beta-\beta_{0}\right|^{2} \gtrsim\left|\beta-\beta_{0}\right|^{2} .
$$

For $A_{2}$, Condition (C.3) yields

$$
\begin{aligned}
A_{2} & \geq P\left(\epsilon_{0} \geq b\right) \int_{a}^{b} P\left(g\left(t-X^{\prime}\left(\beta-\beta_{0}\right)\right)-g_{0}(t)\right)^{2} d \Lambda_{0}(t) \\
& \gtrsim\left\|\zeta(\cdot, \beta)-\zeta_{0}\left(\cdot, \beta_{0}\right)\right\|_{2}^{2} .
\end{aligned}
$$

Therefore

$$
A \lesssim-\left\{\left|\beta-\beta_{0}\right|^{2}+\left\|\zeta(\cdot, \beta)-\zeta_{0}\left(\cdot, \beta_{0}\right)\right\|_{2}^{2}\right\}=-d^{2}\left(\theta, \theta_{0}\right),
$$

and thus from (2.1),

$P l(\beta, \zeta(\cdot, \beta) ; Z)-P l\left(\beta_{0}, \zeta_{0}\left(\cdot, \beta_{0}\right) ; Z\right) \lesssim-d^{2}\left(\theta, \theta_{0}\right)+o\left(d^{2}\left(\theta, \theta_{0}\right)\right) \lesssim-d^{2}\left(\theta, \theta_{0}\right)$, i.e. $P\left(l\left(\theta_{0} ; Z\right)-l(\theta ; Z)\right) \gtrsim d^{2}\left(\theta, \theta_{0}\right)$ for all $\theta \in \Theta_{n}^{p}$, which implies that

$$
\inf _{\left\{d\left(\theta, \theta_{0}\right) \geq \varepsilon, \theta \in \Theta_{n}^{p}\right\}} P\left(l\left(\theta_{0} ; Z\right)-l(\theta ; Z)\right) \gtrsim \varepsilon^{2} .
$$

Hence condition C1 of [3] on page 583 holds with the constant $\alpha=1$ in their notation. 
Next we verify condition C2 of [3]. Denote $\epsilon_{\beta}=Y-X^{\prime} \beta$. It follows that

$$
\begin{aligned}
& {\left[l(\theta ; Z)-l\left(\theta_{0} ; Z\right)\right]^{2}} \\
& =\left\{\Delta g\left(\epsilon_{\beta}\right)-\int_{a}^{b} 1\left(\epsilon_{0} \geq t\right) e^{g\left(t_{\beta}\right)} d t-\Delta g_{0}\left(\epsilon_{0}\right)+\int_{a}^{b} 1\left(\epsilon_{0} \geq t\right) e^{g_{0}(t)} d t\right\}^{2} \\
& \lesssim \Delta\left[g\left(\epsilon_{\beta}\right)-g_{0}\left(\epsilon_{0}\right)\right]^{2}+\left\{\int_{a}^{b} 1\left(\epsilon_{0} \geq t\right)\left[e^{g\left(t_{\beta}\right)}-e^{g_{0}(t)}\right] d t\right\}^{2} \\
& \lesssim \Delta\left[g\left(\epsilon_{\beta}\right)-g_{0}\left(\epsilon_{0}\right)\right]^{2}+\int_{a}^{b}\left[e^{g\left(t_{\beta}\right)}-e^{g_{0}(t)}\right]^{2} d t \\
& \lesssim \Delta\left[g\left(\epsilon_{\beta}\right)-g\left(\epsilon_{0}\right)\right]^{2}+\Delta\left[g\left(\epsilon_{0}\right)-g_{0}\left(\epsilon_{0}\right)\right]^{2} \\
& \quad+\int_{a}^{b}\left[e^{g\left(t_{\beta}\right)}-e^{g(t)}\right]^{2} d t+\int_{a}^{b}\left[e^{g(t)}-e^{g_{0}(t)}\right]^{2} d t \\
& =I_{1}+I_{2}+I_{3}+I_{4},
\end{aligned}
$$

where the second inequality holds because of the Cauchy-Schwartz inequality

$$
\begin{aligned}
& \left\{\int_{a}^{b} 1\left(\epsilon_{0} \geq t\right)\left[e^{g\left(t_{\beta}\right)}-e^{g_{0}(t)}\right] d t\right\}^{2} \\
& \quad \leq\left\{\int_{a}^{b} 1\left(\epsilon_{0} \geq t\right) d t\right\}\left\{\int_{a}^{b}\left[e^{g\left(t_{\beta}\right)}-e^{g_{0}(t)}\right]^{2} d t\right\} \\
& \quad \leq(b-a) \int_{a}^{b}\left[e^{g\left(t_{\beta}\right)}-e^{g_{0}(t)}\right]^{2} d t,
\end{aligned}
$$

and the third inequality holds by subtracting and adding the terms $g\left(\epsilon_{0}\right)$ and $e^{g(t)}$. For $I_{1}$, since $\dot{g} \in \mathcal{G}_{n}^{p-1}$ is bounded, applying the Taylor expansion for $g$ at $\epsilon_{0}$ we obtain

$$
\begin{aligned}
P I_{1} & =P\left\{\Delta\left[g\left(\epsilon_{0}-X^{\prime}\left(\beta-\beta_{0}\right)\right)-g\left(\epsilon_{0}\right)\right]^{2}\right\} \\
& \leq P\left[\dot{g}\left(\epsilon_{0}-X^{\prime}\left(\tilde{\beta}-\beta_{0}\right)\right) X^{\prime}\left(\beta-\beta_{0}\right)\right]^{2} \\
& \lesssim P\left[X^{\prime}\left(\beta-\beta_{0}\right)\right]^{2}=\left(\beta-\beta_{0}\right)^{\prime} P\left(X X^{\prime}\right)\left(\beta-\beta_{0}\right) \\
& \leq \lambda_{d}\left|\beta-\beta_{0}\right|^{2} \lesssim\left|\beta-\beta_{0}\right|^{2},
\end{aligned}
$$

where $\lambda_{d}$ is the largest eigenvalue of $P\left(X X^{\prime}\right)$. For $I_{2}$, since the density function for $(Y, \Delta=1, X)$ is

$$
f_{Y, \Delta, X}(y, 1, x)=\lambda_{0}\left(y-x^{\prime} \beta_{0}\right) e^{-\Lambda_{0}\left(y-x^{\prime} \beta_{0}\right)} \bar{G}_{C \mid X}(y \mid X=x) f_{X}(x),
$$


it follows that

$$
\begin{aligned}
P I_{2} & =P\left[\Delta\left(g-g_{0}\right)^{2}\left(\epsilon_{0}\right)\right] \\
& =\int_{\mathcal{X}}\left\{\int_{a}^{b}\left(g(t)-g_{0}(t)\right)^{2} \lambda_{0}(t) e^{-\Lambda_{0}(t)} \bar{G}_{C \mid X}\left(t+x^{\prime} \beta_{0} \mid X=x\right) d t\right\} \\
& \leq \int_{\mathcal{X}}\left\{\int_{a}^{b}\left(g(t)-g_{0}(t)\right)^{2} d \Lambda_{0}(t)\right\} f_{X}(x) d x \\
& =\left\|\zeta\left(\cdot, \beta_{0}\right)-\zeta_{0}\left(\cdot, \beta_{0}\right)\right\|_{2}^{2} .
\end{aligned}
$$

Then for $I_{3}$, since $g \in \mathcal{G}_{n}^{p}$ is bounded, it follows that

$$
\begin{aligned}
P I_{3} & =P \int_{a}^{b}\left[e^{g\left(t-X^{\prime}\left(\beta-\beta_{0}\right)\right)}-e^{g(t)}\right]^{2} d t \\
& =P \int_{a}^{b} e^{2 g\left(t-X^{\prime}\left(\tilde{\beta}-\beta_{0}\right)\right)}\left[X^{\prime}\left(\beta-\beta_{0}\right)\right]^{2} d t \\
& \lesssim \int_{a}^{b} P\left[X^{\prime}\left(\beta-\beta_{0}\right)\right]^{2} d t \\
& \lesssim\left(\beta-\beta_{0}\right)^{\prime} P\left[X X^{\prime}\right]\left(\beta-\beta_{0}\right) \lesssim\left|\beta-\beta_{0}\right|^{2} .
\end{aligned}
$$

Finally for $I_{4}$, by the Taylor expansion for $e^{g(t)}$ at $g_{0}$, we have

$$
\begin{aligned}
P I_{4} & =\int_{a}^{b}\left[e^{g(t)}-e^{g_{0}(t)}\right]^{2} d t \\
& \leq \int_{a}^{b} e^{2 \tilde{g}(t)}\left(g(t)-g_{0}(t)\right)^{2} d t \\
& =\int_{a}^{b} e^{2 \tilde{g}(t)-g_{0}(t)}\left(g(t)-g_{0}(t)\right)^{2} d \Lambda_{0}(t) \\
& \lesssim \int_{a}^{b}\left(g(t)-g_{0}(t)\right)^{2} d \Lambda_{0}(t)=\left\|\zeta\left(\cdot, \beta_{0}\right)-\zeta_{0}\left(\cdot, \beta_{0}\right)\right\|_{2}^{2},
\end{aligned}
$$

where $\tilde{g}(t)=g_{0}(t)+\xi\left(g-g_{0}\right)(t)$ for some $0<\xi<1$ and hence is bounded. Since $\left\|\zeta\left(\cdot, \beta_{0}\right)-\zeta_{0}\left(\cdot, \beta_{0}\right)\right\|_{2} \leq \mid \zeta(\cdot, \beta)-\zeta\left(\cdot, \beta_{0}\right)\left\|_{2}+\right\| \zeta(\cdot, \beta)-\zeta_{0}\left(\cdot, \beta_{0}\right) \|_{2} \lesssim$ $\left|\beta-\beta_{0}\right|+\left\|\zeta(\cdot, \beta)-\zeta_{0}\left(\cdot, \beta_{0}\right)\right\|_{2}$, we have

$$
P\left(l(\theta ; Z)-l\left(\theta_{0} ; Z\right)\right)^{2} \lesssim\left|\beta-\beta_{0}\right|^{2}+\left\|\zeta(\cdot, \beta)-\zeta_{0}\left(\cdot, \beta_{0}\right)\right\|_{2}^{2}=d^{2}\left(\theta, \theta_{0}\right)
$$

for any $\theta \in \Theta_{n}^{p}$, which implies that

$$
\begin{aligned}
\sup _{\left\{d\left(\theta, \theta_{0}\right) \leq \varepsilon, \theta \in \Theta_{n}^{p}\right\}} \operatorname{Var}\left(l\left(\theta_{0} ; Z\right)-l(\theta ; Z)\right) & \\
& \leq \sup _{\left\{d\left(\theta, \theta_{0}\right) \leq \varepsilon, \theta \in \Theta_{n}^{p}\right\}} P\left(l\left(\theta_{0} ; Z\right)-l(\theta ; Z)\right)^{2} \lesssim \varepsilon^{2} .
\end{aligned}
$$


So condition $\mathrm{C} 2$ of [3] on page 583 holds with the constant $\beta=1$ in their notation.

Finally, we verify condition C3 of [3]. By lemma 7.3, for $\mathcal{F}_{n}=\{l(\theta ; Z)-$ $\left.l\left(\theta_{0, n} ; Z\right): \theta \in \Theta_{n}^{p}\right\}$, we have $N_{[]}\left(\varepsilon, \mathcal{F}_{n},\|\cdot\|_{\infty}\right) \lesssim(1 / \varepsilon)^{c q_{n}+d}$. Then by the fact that the covering number is bounded by the bracketing number, it follows that

$$
H\left(\varepsilon, \mathcal{F}_{n},\|\cdot\|_{\infty}\right)=\log N\left(\varepsilon, \mathcal{F}_{n},\|\cdot\|_{\infty}\right) \lesssim\left(c q_{n}+d\right) \log (1 / \varepsilon) \lesssim n^{\nu} \log (1 / \varepsilon) .
$$

So condition C3 of [3] on page 583 holds with the constants $2 r_{0}=\nu$ and $r=0^{+}$in their notation.

Therefore, the constant $\tau$ in Theorem 1 of [3] on page 584 is $\frac{1-\nu}{2}-\frac{\log \log n}{2 \log n}$. Since $\frac{\log \log n}{2 \log n} \rightarrow 0$ as $n \rightarrow 0$, we can pick a $\tilde{\nu}$ slightly greater than $\nu$ such that $\frac{1-\tilde{\nu}}{2} \leq \frac{1-\nu}{2}-\frac{\log \log n}{2 \log n}$ for $n$ large. We still denote $\tilde{\nu}$ by $\nu$ and then $\tau=\frac{1-\nu}{2}$. Since $\hat{\theta}_{n}$ maximizes the empirical log-likelihood $\mathbb{P}_{n} l(\theta ; Z)$ over the sieve space $\Theta_{n}^{p}$, we have that $\hat{\theta}_{n}$ satisfies inequality (1.1) in [3] with $\eta_{n}=0$. By Lemma 7.2 , there exists an $\zeta_{0, n}\left(\cdot, \beta_{0}\right) \in \mathcal{H}_{n}^{p}$ such that $\left\|\zeta_{0, n}\left(\cdot, \beta_{0}\right)-\zeta_{0}\left(\cdot, \beta_{0}\right)\right\|_{\infty}=O\left(n^{-p \nu}\right)$. Moreover, by the Taylor expansion for $P\left[l\left(\beta_{0}, \zeta_{0}\left(\cdot, \beta_{0}\right) ; Z\right)-l(\beta, \zeta(\cdot, \beta) ; Z)\right]$ in $(2.1)$ and plugging in $\theta=\theta_{0, n}=$ $\left(\beta_{0}, \zeta_{0, n}\left(\cdot, \beta_{0}\right)\right)$, the Kullback-Leibler distance between $\theta_{0, n}=\left(\beta_{0}, \zeta_{0, n}\left(\cdot, \beta_{0}\right)\right)$ and $\theta_{0}=\left(\beta_{0}, \zeta_{0}\left(\cdot, \beta_{0}\right)\right)$ is given as

$$
\begin{aligned}
& K\left(\theta_{0, n}, \theta_{0}\right) \\
&= P\left[l\left(\theta_{0} ; Z\right)-l\left(\theta_{0, n} ; Z\right)\right] \\
&=\quad P\left\{\ddot{l}_{\zeta \zeta}\left(\beta_{0}, \zeta_{0}\left(\cdot, \beta_{0}\right) ; Z\right)\left[\zeta_{0, n}\left(\cdot, \beta_{0}\right)-\zeta_{0}\left(\cdot, \beta_{0}\right), \zeta_{0, n}\left(\cdot, \beta_{0}\right)-\zeta_{0}\left(\cdot, \beta_{0}\right)\right]\right\} \\
&+o\left(\left\|\zeta_{0, n}\left(\cdot, \beta_{0}\right)-\zeta_{0}\left(\cdot, \beta_{0}\right)\right\|_{2}^{2}\right) \\
&=\quad P\left\{\int_{a}^{b} 1\left(\epsilon_{0} \geq t\right) \exp \left\{g_{0}(t)\right\}\left(g_{0, n}(t)-g_{0}(t)\right)^{2} d t\right\} \\
& \quad+o\left(\left\|\zeta_{0, n}\left(\cdot, \beta_{0}\right)-\zeta_{0}\left(\cdot, \beta_{0}\right)\right\|_{2}^{2}\right) \\
& \leq \quad \int_{a}^{b}\left(g_{0, n}(t)-g_{0}(t)\right)^{2} d \Lambda_{0}(t)+o\left(\left\|\zeta_{0, n}\left(\cdot, \beta_{0}\right)-\zeta_{0}\left(\cdot, \beta_{0}\right)\right\|_{2}^{2}\right) \\
&=\left\|\zeta_{0, n}\left(\cdot, \beta_{0}\right)-\zeta_{0}\left(\cdot, \beta_{0}\right)\right\|_{2}^{2}+o\left(\left\|\zeta_{0, n}\left(\cdot, \beta_{0}\right)-\zeta_{0}\left(\cdot, \beta_{0}\right)\right\|_{2}^{2}\right)=O\left(n^{-2 p \nu}\right),
\end{aligned}
$$

where the last equality holds because $\left\|\zeta_{0, n}\left(\cdot, \beta_{0}\right)-\zeta_{0}\left(\cdot, \beta_{0}\right)\right\|_{2} \leq \| \zeta_{0, n}\left(\cdot, \beta_{0}\right)-$ $\zeta_{0}\left(\cdot, \beta_{0}\right) \|_{\infty}=O\left(n^{-p \nu}\right)$. Therefore $K^{1 / 2}\left(\theta_{0, n}, \theta_{0}\right)=O\left(n^{-p \nu}\right)$. Thus by Theorem 1 of [3], we obtain the convergence rate for $\hat{\theta}_{n}$ as follows

$$
d\left(\hat{\theta}_{n}, \theta_{0}\right)=O_{p}\left\{\max \left(n^{-(1-\nu) / 2}, n^{-p \nu}, n^{-p \nu}\right)\right\}=O_{p}\left\{n^{-\min (p \nu,(1-\nu) / 2)}\right\} .
$$


3. Proof of Theorem 4.3. Define $\mathbf{w}_{n}^{*}(t)=-\dot{\hat{g}}_{n}(t) \bar{X}\left(t ; \hat{\beta}_{n}\right)$. Then we have

$$
l_{\hat{\beta}_{n}}^{*}(Y, \Delta, X)=\dot{l}_{\beta}\left(\hat{\theta}_{n} ; Z\right)-\dot{l}_{\zeta}\left(\hat{\theta}_{n} ; Z\right)\left[\mathbf{h}_{n}^{*}\right] .
$$

Define

$$
\begin{aligned}
I^{j k}\left(\beta_{0}\right)=P[ & \left\{i_{\beta_{j}}\left(\theta_{0} ; Z\right)-i_{\zeta}\left(\theta_{0} ; Z\right)\left[h_{j}^{*}\right]\right\} \\
& \left.\times\left\{i_{\beta_{k}}\left(\theta_{0} ; Z\right)-i_{\zeta}\left(\theta_{0} ; Z\right)\left[h_{k}^{*}\right]\right\}\right] \equiv P A^{j k}\left(\theta_{0} ; Z\right), \\
\hat{I}_{n}^{j k}\left(\hat{\beta}_{n}\right)=\mathbb{P}_{n}[ & \left\{i_{\beta_{j}}\left(\hat{\theta}_{n} ; Z\right)-i_{\zeta}\left(\hat{\theta}_{n} ; Z\right)\left[h_{j, n}^{*}\right]\right\} \\
& \left.\times\left\{i_{\beta_{k}}\left(\hat{\theta}_{n} ; Z\right)-i_{\zeta}\left(\hat{\theta}_{n} ; Z\right)\left[h_{k, n}^{*}\right]\right\}\right] \equiv \mathbb{P}_{n} A_{n}^{j k}\left(\hat{\theta}_{n} ; Z\right),
\end{aligned}
$$

where $h_{j}^{*}$ is defined in Lemma 7.4, see also Equation (7.1) in the main text. We will prove $\mathbb{P}_{n} A_{n}^{j k}\left(\hat{\theta}_{n} ; Z\right) \rightarrow P A^{j k}\left(\theta_{0} ; Z\right)$ in probability for all $j, k=$ $1, \ldots, d$. Let

$$
\begin{aligned}
\mathbb{P}_{n} A_{n}^{j k}\left(\hat{\theta}_{n} ; Z\right)-P A^{j k}\left(\theta_{0} ; Z\right)= & \left(\mathbb{P}_{n}-P\right) A_{n}^{j k}\left(\hat{\theta}_{n} ; Z\right) \\
& \quad+P\left\{A_{n}^{j k}\left(\hat{\theta}_{n} ; Z\right)-A^{j k}\left(\theta_{0} ; Z\right)\right\} \\
= & I_{1 n}+I_{2 n} .
\end{aligned}
$$

For $I_{1 n}$, we first define the class of functions

$$
\begin{array}{r}
\mathcal{F}_{n, j}^{\beta, \zeta}(\eta)=\left\{i_{\beta_{j}}(\theta ; z)-\dot{l}_{\zeta}(\theta ; z)\left[h_{j}\right]: \theta \in \Theta_{n}^{p}, d\left(\theta, \theta_{0}\right) \leq \eta, h_{j} \in \mathcal{H}_{n}^{2},\right. \\
\left.\left\|\dot{g}(\psi(\cdot, \beta))-\dot{g}_{0}\left(\psi\left(\cdot, \beta_{0}\right)\right)\right\|_{2} \leq \eta,\left\|h_{j}-h_{j}^{*}\right\|_{\infty} \leq \eta\right\} .
\end{array}
$$

Then by Lemmas 7.5 and 7.6 we have

$$
N_{[]}\left(\varepsilon, \mathcal{F}_{n, j}^{\beta, \zeta},\|\cdot\|_{\infty}\right) \lesssim(1 / \varepsilon)^{c q_{n}+d}
$$

for some constant $c>0$. This is because for any function $\dot{l}_{\beta_{j}}(\theta ; z)-\dot{l}_{\zeta}(\theta ; z)\left[h_{j}\right] \in$ $\mathcal{F}_{n, j}^{\beta, \zeta}(\eta)$, it can be written as

$$
\begin{aligned}
i_{\beta_{j}}(\theta ; z)- & \dot{l}_{\zeta}(\theta ; z)\left[h_{j}\right] \\
= & \left\{i_{\beta_{j}}(\theta ; z)-i_{\beta_{j}}\left(\theta_{0} ; z\right)\right\}-\left\{i_{\zeta}(\theta ; z)\left[h_{j}^{*}\right]-i_{\zeta}\left(\theta_{0} ; z\right)\left[h_{j}^{*}\right]\right\} \\
& \quad+\left\{i_{\zeta}(\theta ; z)\left[h_{j}^{*}\right]-\dot{i}_{\zeta}(\theta ; z)\left[h_{j}\right]\right\}+\left\{i_{\beta_{j}}\left(\theta_{0} ; z\right)-i_{\zeta}\left(\theta_{0} ; z\right)\left[h_{j}^{*}\right]\right\} \\
= & A_{1}+A_{2}+A_{3}+A_{4}
\end{aligned}
$$

where $A_{1} \in \mathcal{F}_{n, j}^{\beta}(\eta)$ and $A_{2} \in \mathcal{F}_{n, j}^{\zeta}(\eta)$ defined in Lemma 7.6, $A_{3} \in \mathcal{F}_{n}^{j}(\eta)$ defined in Lemma 7.5, and $A_{4}$ is a fixed function (the efficient score function). 
Assume $A_{m}^{L} \leq A_{m} \leq A_{m}^{U}$ with $\left\|A_{m}^{U}-A_{m}^{L}\right\|_{\infty} \lesssim \varepsilon, m=1,2,3$. Then $A_{m}^{L}+$ $A_{m^{\prime}}^{L} \leq A_{m}+A_{m^{\prime}} \leq A_{m}^{U}+A_{m^{\prime}}^{U}$ with $\left\|\left(A_{m}^{U}+A_{m^{\prime}}^{U}\right)-\left(A_{m}^{L}+A_{m^{\prime}}^{L}\right)\right\|_{\infty} \leq$ $\left\|A_{m}^{U}-A_{m}^{L}\right\|_{\infty}+\left\|A_{m^{\prime}}^{U}-A_{m^{\prime}}^{L}\right\|_{\infty} \lesssim \varepsilon$. Therefore the $\varepsilon$-bracketing number associated with $\|\cdot\|_{\infty}$ for $\mathcal{F}_{n, j}^{\beta, \zeta}(\eta)$ is also bounded by $(\eta / \varepsilon)^{c q_{n}+\alpha}$.

We next define the class of functions

$$
\begin{gathered}
\mathcal{F}_{n, j k}^{\beta, \zeta}(\eta)=\left\{\left(i_{\beta_{j}}(\theta ; z)-i_{\zeta}(\theta ; z)\left[h_{j}\right]\right)\left(i_{\beta_{k}}(\theta ; z)-i_{\zeta}(\theta ; z)\left[h_{k}\right]\right): \theta \in \Theta_{n}^{p},\right. \\
h_{j}, h_{k} \in \mathcal{H}_{n}^{p-1}, d\left(\theta, \theta_{0}\right) \leq \eta,\left\|\dot{g}(\psi(\cdot, \beta))-\dot{g}_{0}\left(\psi\left(\cdot, \beta_{0}\right)\right)\right\|_{2} \leq \eta, \\
\left.\left\|h_{j}-h_{j}^{*}\right\|_{\infty} \leq \eta,\left\|h_{k}-h_{k}^{*}\right\|_{\infty} \leq \eta\right\} .
\end{gathered}
$$

Then if $B_{j}^{L} \leq i_{\beta_{j}}(\theta ; z)-i_{\zeta}(\theta ; z)\left[h_{j}\right] \leq B_{j}^{U}$ and $B_{k}^{L} \leq i_{\beta_{j}}(\theta ; z)-i_{\zeta}(\theta ; z)\left[h_{k}\right] \leq$ $B_{k}^{U}$ with $\left\|B_{j}^{U}-B_{j}^{L}\right\|_{\infty} \leq \varepsilon$ and $\left\|B_{k}^{U}-B_{k}^{L}\right\|_{\infty} \leq \varepsilon$, we have $B_{j}^{*} B_{k}^{*} \leq\left(i_{\beta_{j}}(\theta ; z)-\right.$ $\left.i_{\zeta}(\theta ; z)\left[h_{j}\right]\right)\left(i_{\beta_{k}}(\theta ; z)-\dot{l}_{\zeta}(\theta ; z)\left[h_{k}\right]\right) \leq B_{j}^{* *} B_{k}^{* *}$, where $B_{j}^{*}, B_{j}^{* *}$ take values of either $B_{j}^{L}$ or $B_{j}^{U}$, and the same for $B_{k}^{*}, B_{k}^{* *}$. Thus

$$
\begin{aligned}
\left\|B_{j}^{* *} B_{k}^{* *}-B_{j}^{*} B_{k}^{*}\right\|_{\infty} & =\left\|\left(B_{j}^{* *}-B_{j}^{*}\right) B_{k}^{* *}+\left(B_{k}^{* *}-B_{k}^{*}\right) B_{j}^{*}\right\|_{\infty} \\
& =\left\|B_{j}^{* *}-B_{j}^{*}\right\|_{\infty}\left\|B_{k}^{* *}\right\|_{\infty}+\left\|B_{k}^{* *}-B_{k}^{*}\right\|_{\infty}\left\|_{j}^{*}\right\|_{\infty} \\
& \lesssim\left\|B_{j}^{U}-B_{j}^{L}\right\|_{\infty}+\left\|B_{k}^{U}-B_{k}^{L}\right\|_{\infty} \\
& \lesssim \varepsilon,
\end{aligned}
$$

which yields

$$
N_{[]}\left(\varepsilon, \mathcal{F}_{n, j k}^{\beta, \zeta},\|\cdot\|_{\infty}\right) \lesssim(1 / \varepsilon)^{c q_{n}+d}
$$

for some constant $c>0$.

Finally, similar to the verification of Assumption (A4) in the proof of Theorem 4.2 and together with the following fact:

$$
\begin{aligned}
& \left\|h_{j}^{*}\left(\cdot, \beta_{0}\right)-h_{j, n}^{*}\left(\cdot, \hat{\beta}_{n}\right)\right\|_{\infty} \\
& \quad=\left\|\dot{g}_{0}(t) P\left(X \mid \epsilon_{0} \geq t\right)-\dot{\hat{g}}_{n}\left(t_{\hat{\beta}_{n}}\right) \bar{X}\left(t_{\hat{\beta}_{n}} ; \hat{\beta}_{n}\right)\right\|_{\infty} \\
& \quad \leq\left\|\dot{g}_{0}(t) P\left(X \mid \epsilon_{0} \geq t\right)-\dot{\hat{g}}_{n}(t) \bar{X}\left(t ; \hat{\beta}_{n}\right)\right\|_{\infty} \\
& \quad+\left\|\dot{\hat{g}}_{n}\left(t_{\hat{\beta}_{n}}\right) \bar{X}\left(t_{\hat{\beta}_{n}} ; \hat{\beta}_{n}\right)-\dot{\hat{g}}_{n}\left(t_{\hat{\beta}_{n}}\right) \bar{X}\left(t ; \hat{\beta}_{n}\right)\right\|_{\infty} \\
& \quad+\left\|\dot{\hat{g}}_{n}\left(t_{\hat{\beta}_{n}}\right) \bar{X}\left(t ; \hat{\beta}_{n}\right)-\dot{\hat{g}}_{n}(t) \bar{X}\left(t ; \hat{\beta}_{n}\right)\right\|_{\infty},
\end{aligned}
$$

where the first term on the right hand side of inequality (3.1) is

$$
\begin{gathered}
\left\|\dot{g}_{0}(t) P\left(X \mid \epsilon_{0} \geq t\right)-\dot{\hat{g}}_{n}(t) \bar{X}\left(t ; \hat{\beta}_{n}\right)\right\|_{\infty} \\
\leq\left\|\dot{g}_{0}(t)-\dot{\hat{g}}_{n}(t)\right\|_{\infty}\left\|P\left(X \mid \epsilon_{0} \geq t\right)\right\|_{\infty} \\
\quad+\left\|P\left(X \mid \epsilon_{0} \geq t\right)-\bar{X}\left(t ; \hat{\beta}_{n}\right)\right\|_{\infty}\left\|\dot{\hat{g}}_{n}(t)\right\|_{\infty} \\
=O_{p}\left(n^{-2 v}\right)+O_{p}\left(n^{-1 / 2}\right)=O_{p}\left(n^{-2 v}\right)
\end{gathered}
$$


by Lemma 7.4 and Corollary 6.21 in [2] for the first term and straightforward argument using empirical process theory for Donsker classes for the second term, together with the boundedness of $\left\|P\left(X \mid \epsilon_{0} \geq t\right)\right\|_{\infty}$ and $\left\|\dot{\hat{g}}_{n}(t)\right\|_{\infty}$, and it is straightforward to see that the remaining two terms on the right hand side of inequality (3.1) is $O_{p}\left(n^{-1 / 2}\right)$. Thus we have $I_{1 n}=o_{p}(1)$.

That $I_{2 n}=o_{p}(1)$ can be argued directly by the dominated convergence theorem. We now have proved the theorem.

\section{References.}

[1] Ding, Y. (2010). Some New Insights about the Accelerated Failure Time Model. Ph.D. Thesis, Biostatistics, University of Michigan.

[2] Schumaker, L. (1981). Spline Functions: Basic Theory. Wiley, New York.

[3] Shen, X. and Wong, W. H. (1994). Convergence Rate of Sieve Estimates. The Annals of Statistics 22 580-615.

[4] Wellner, J. A. and Zhang, Y. (2007). Two Likelihood-based Semiparametric Estimation Methods for Panel Count Data with Covariates. The Annals of Statistics 35 $2106-2142$.

Department of Biostatistics UNIVERSITY OF MiCHIGAN 1420 WASHINGTON Heights ANN ARBor, MI 48109-2029

E-MAIL: yingding@umich.edu bnan@umich.edu 